\title{
Pattern of Paediatric Brain Tumours Evaluated in BSMMU, Dhaka, Bangladesh
}

\author{
FAUZIA JAHAN ${ }^{1}$, MOHAMMED KAMAL ${ }^{2}$, SHAMSUNNAHAR SULTANA ${ }^{3}$
}

\begin{abstract}
:
Background: In children Central Nervous System (CNS) is the second most common site for primary tumours superseded only by leukaemias. Paediatric brain tumours differ from adult one by their type, location and outcome.

Objectives: To study the frequency and histomorphological spectrum of CNS tumours in Children and to correlate with age, sex and distribution of tumours.

Methodology: This study was carried out at the Department of Pathology, Bangabandhu Shiekh Mujib Medical University (BSMMU) Dhaka during the period of July 2006 to June 2007. Samples from all Paediatric age and both sexes were included in this study.

Results: We studied 55 cases of Paediatric CNS tumours. The patients' age ranged between 2 months to 15 years. The mean age at diagnosis was 10.12 \pm 4.18 years. Regarding location $59 \%$ was infratentorial and $41 \%$ were supratentorial. Most common tumour was medulloblastoma (23\%). Twenty Seven (27\%) of the tumours were high grade.
\end{abstract}

Key words: Paediatric brain tumour, medulloblastoma, infratentorial location.

\section{Introduction}

Tumours of the Central Nervous System (CNS) are a heterogeneous group of neoplasms comprising all forms of primary neoplasms derived from craniovertebral structures and also those metastasizing to this location. Intracranial neoplasms represent the 6th most common form of neoplasm in adults. In children the CNS is the second most common site for primary tumours superseded only by leukaemia ${ }^{1}$.

Clinical information that are very important and aid in the differential histological diagnoses of tumours of central neuraxis are age and sex of the patient and anatomic location of the lesion. Actually no age is immune to the occurrence of intracranial tumour. There are peak in the occurrence of various tumours. Children and adults have different distribution of subtypes of CNS tumours; location also varies in these age groups ${ }^{2}$.

1. Assistant Professor, Department of Pathology, Bangladesh Medical College, Dhaka

2. Chairman and Professor, Department of Pathology, Bangabandhu Sheikh Mujib Medical University (BSMMU). Dhaka

3. Assistant Professor, Department of Pathology, Holy family Red Cresent Medical College, Dhaka

Correspondence: Dr. Fauzia Jahan, Cell: 01715-254067, E-mail: fauzia0610@gmail.com
Primary Paediatric brain tumours are more common in the first decade and highest in children under 8 years of age. Neoplasms in children less than 2 years of age represent a distinctly different histologic spectrum compared to older children. Sixty percent tumours in children below one year age are supratentorial and $40 \%$ are infratentorial. The percentage reverses after one year of age in favor of posterior fossa ${ }^{3}$. Commonest brain tumours in this age group (<1 year) include astrocytoma, ependymoma and medulloblastoma. In older children approximately half of the intracranial neoplasms are gliomas followed by medulloblastoma, ependymoma and craniopharyngioma ${ }^{4}$.

Paediatric brain tumour differs from adult one in several ways.

Firstly, the types of tumour occurring in children are uncommon in adults and vice versa. Secondly, tumours in posterior fossa mostly occur in children compared to adults. Thirdly, the value of extensive tumour resection for different tumours is confirmed in children, which are still controversial for adult tumors. Fourthly chemotherapy plays a greater role in improving overall out come for Paediatric tumors than in adults. It is also seen that high-grade and 
incompletely resected low-grade tumor in children under 3 years of age are given chemotherapy to avoid radiotherapy and morbidity caused by it. Finally prognoses of histologically similar tumors are better in children than adults ${ }^{5}$. Formalin fixation with routine processing, paraffin embedding and H\&E staining are the preferred method for routine initial analysis which gives the surgical neuropathologist most of the information needed for a precise diagnosis in most cases $^{6}$.

\section{Methodology}

This study was carried out at the Department of Pathology, Bangabandhu Shiekh Mujib Medical University (BSMMU), Dhaka with an aim to see the histological pattern, distribution of CNS tumours of children and to correlate with age and sex during the period of July 2006 to June 2007. Samples of children below 15 years were collected and both sexes were included in this study.

A total of 55 samples of central nervous system tumours were collected from Department of Pathology, BSMMU, Dhaka Medical College Hospital, private laboratories of Dhaka city(where samples are sent from different regions of Bangladesh), Medical colleges of Chittagong, Rajshahi, Mymensingh and Sylhet. Hematoxylin and Eosin Stained slides and blocks were collected from above mentioned institutes along with information regarding age, sex. Location of tumours were detected in all institutes by imaging studies and per operative findings. Informations were also collected about initial histological diagnosis made by them. In BSMMU, fresh specimens of different brain tumour of children from Neurosurgery Department were collected after biopsy or excision. After collection samples were preserved in formalin. Diagnoses in all cases from BSMMU and as well as from other institutes were made on Hematoxylin and Eosin stained sections based on standard histomorphological features. For arriving at a correct diagnosis, routinely stained sections were first examined under low and later under high magnification by author and later confirmed by expert histopathologist.

For CNS classification of tumour in children WHO method was followed? ${ }^{7}$.
Result:

Age and sex of the study cases

Patients included in this study were 55 children $(<15$ years). The age range of Paediatric group was from 2 months to 15 years. The mean age at diagnosis was $10.12 \pm 4.18$ years. Out of total 55 cases 37 were males and 18 were females with male to female ratio of 2.1:1.

\section{Location of the tumour:}

In this study out of total 55 cases 47 were intracranial tumours, 5 spinal tumours and rest 3 where site were not mentioned (Table:1). Out of 47 intracranial tumours 28 tumours were located in infratentorial region and 19 within supratentorial region (Table: 2). Predominant site was posterior fossa (21 cases), followed by sellar/ suprasellar (6 cases) and Cerebello-Pontine (C-P) angle ( 5 cases) (Table-III).

Table-I

Distribution of tumour according to site

\begin{tabular}{llc}
\hline Site & Number of tumours \\
\hline Intracranial & Supratentorial & 19 \\
& Infratentorial & 28 \\
Spinal & 05 \\
Site not mentioned & 03 \\
\hline
\end{tabular}

Table-II

Distribution of intracranial tumour according to site

\begin{tabular}{llc}
\hline Site & & Number of tumours \\
\hline Supratentorial & Ventricles & 03 \\
& Frontal & 01 \\
& Parietal & 01 \\
& Occipital & 02 \\
& Parieto-occipital & 02 \\
& Temporal & 01 \\
& Temperoparietal & 01 \\
& Sellar/Suprasellar region & 06 \\
& Thalamus & 02 \\
Infratentorial & Post fossa & 21 \\
& C-P angle & 05 \\
& Cerebellum & 01 \\
& Pons & 01 \\
\hline
\end{tabular}


Table-III

Tumours in predominant site

\begin{tabular}{llc}
\hline Site & Tumours & No of tumours \\
\hline Posterior fossa & Medulloblastoma & 12 \\
& Pilocytic astrocytoma & 06 \\
& Fibrillary astrocytoma & 09 \\
& Ependymoma & 09 \\
& Anaplastic astrocytoma & 01 \\
Sellar/suprasellar & Craniopharyngioma & 03 \\
region & Pilocytic astrocytoma & 03 \\
C-P angle & Schwannoma & 09 \\
\hline
\end{tabular}

\section{Histological types of tumour:}

The most commonly encountered tumours in this age group was medulloblastoma (12 cases) (Fig:2) followed by ependymoma ( 9 cases), fibrillary astrocytoma ( 9 cases), schwannoma (9 cases), pilocytic astrocytoma (6 cases), 3 cases of craniopharyngioma and one case each of anaplastic astrocytoma, premitive neuroectodermal tumour (PNET), choroid plexus papilloma, oligodendroglioma, glioblastoma, ganglioglioma and metastatic ganglioneuroblastoma (Fig:1).

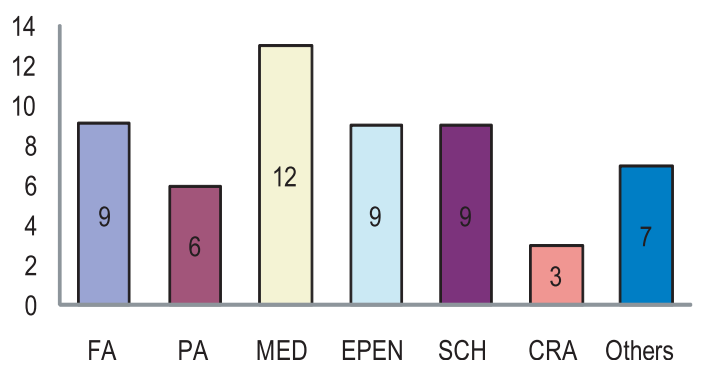

FA:Fibrillary astrocytoma, PA:Pilocytic astrocytoma, MED:Medulloblastoma EPEN:Ependymoma, SCH:Schwannoma, CRA: craniopharyngioma

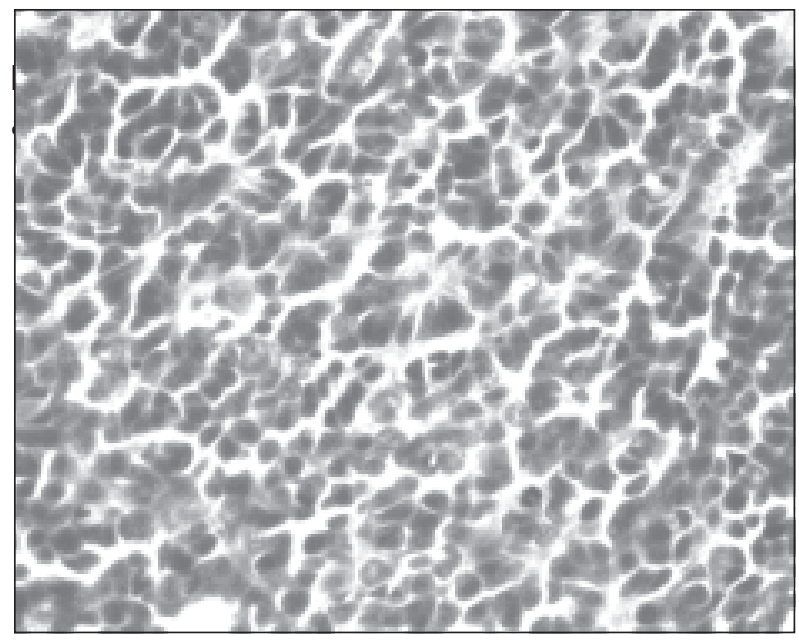

Fig.-2: Photomicrograph of medulobastoma showing small round cells with neuroblastic rosettes (H\& E stain)

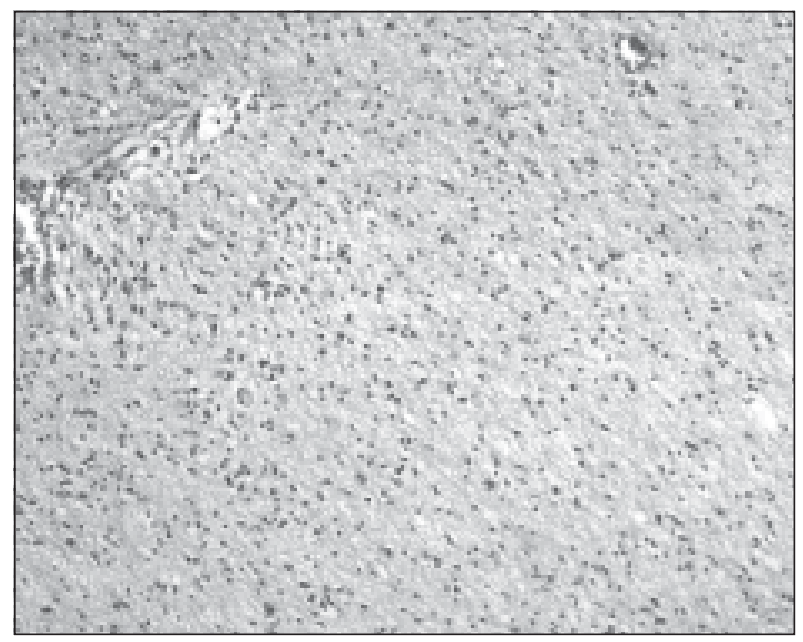

Fig.-3: Photomicrograph of fibrillary astrocytoma showing low cellularity and mild nuclear atypia (H\&E stain)

\section{Tumour grade:}

Out of 55 cases 15 cases were high grade lesion. These were medulloblastoma (12 =grade IV), and one each case of anaplastic astrocytoma (grade III), glioblastoma (grade IV) and PNET (grade IV). The rest were low grade (Grade I and II) tumours. Grade II lesions included ependymomas and fibrillary astrocytomas.

\section{Discussion}

Though childhood or paediatric age groups have been quoted upto 18 years $^{8}$, the Government Hospitals in Bangladesh and BSMMU admit children upto 15 years in the paediatric wards ${ }^{9}$. Therefore for working definition Paediatric age group is taken as children upto 15 years of age in this study.

The current study was designed to segregate Paediatric brain tumors from adult tumors and to determine the tumour site and morphologic patterns. Brain tumors are the second most common solid tumors that affect children of all ages, ethnicities and races, albeit with variations.

WHO guidelines were followed for histological classification of all Paediatric CNS tumours

In general the males are slightly more affected than female ${ }^{10}$. The present study analyzed 55 cases of Paediatric CNS tumours with the male to female ratio of 2.1:1

The mean age for all cases in Karachi was 8.8 years, with a marginal variation for malignancies arising in the cerebrum and cerebellum. This is higher than the mean or median age reported by other authors. The 
median age at diagnosis was 6 years with a male-tofemale ratio of 1.3:1, in a published study (11). This present study showed a mean age to be 10.12 years, which is higher than the studies carried out in different series. This variation may likely be due to differences in the study size and selection of cases.

In children $70 \%$ of brain tumors are infratentorial and $30 \%$ are supratentorial whereas it is reverse in adults $^{12}$. Posterior fossa tumors comprise a great number of childhood brain tumors ${ }^{13}$. In this study common location was infratentorial region (59.5\%) and posterior fossa being the predominant site.

Intracranial neoplasms account for $20-20 \%$ of all Paediatric malignancies of which medulloblastoma are most frequently encountered ${ }^{14}$. Compared to the previously published studies our series also has a higher number of medulloblastoma.. It was also found to be the commonest posterior fossa tumour in children included in our study accounting for $21 \%$ (12/ 55). Out of 12 cases of medulloblastoma 10 occurred in posterior cranial fossa and rest two in cerebellum and pons in this present series.

The tumors in Paediatric age group in the current study ( 55 cases) was dominated by medulloblastoma, is followed by Ependymoma (9cases $=16.07 \%$ ), fibrillary astrocytoma (9cases $=16.07 \%$ ), schwannoma ( 9 cases $=16.07 \%$ ) pilocytic astrocytomas ( 6 cases $=10.71 \%), 3$ cases of craniopharyngiomas and single cases of anaplastic astrocytoma, PNET, choroid plexus papilloma, oligodendroglioma, ganglioglioma, and metastatic ganglioneuroblastoma.

Six completely resectable cases of pilocytic astrocytoma (grade I) were encountered in this study.

\section{Conclusion:}

The results of these studies show medulloblastoma to be the most common Paediatric brain tumour. Males were more commonly affected than females. Posterior cranial fossa was the most common site.

\section{References:}

1. Visudhiphan P, Chiemchanya S, Dheandhanoo D. Brain tumors in children at Ramathibodi Hospital. J Med Assoc Thai 1989; 72(1):102-8.

2. Teddy P. J. Intracranial tumors. In: John GG, Warrel DA, editors. Concise Oxford Textbook of Medicine, $3^{\text {rd }}$ edn. Edinburgh: Oxford University Press; 2000. P. 1336.

3. Lellouch-Tubiana A,Pfister A, Da Lage C. Histologioc classification and prevalence of tumours of the central nervous system in infants. Ann Pathol 1998;9(3):195-98.

4. Osborne AG, Rauschning W. Brain Tumors and Tumor like Masses: Classification and Differential Diagnosis. Diagnostic Neuroradiology.1 ${ }^{\text {st }}$ edition. Missouri: Mosby St Louis; 1994. p .401-10.

5. Pollack IF. Paediatric brain tumors.Semin, Surg, Oncol 1999; 16: 73-90.

6. Douglas C. Miller. Modern Surgical Neuropathology. Cambridge University Press; 2009. p.1-9.

7. Kleihues P., Burger P. C, Scheithauer B. W. The new WHO classification of brain tumors. Brain Pathlo 1993; 3(3) : 255-68.

8. Richard EB, Robert MK, Hal BJ. Nelson text book of Paediatric, $17^{\text {th }}$ edition. Philadelphia; Saunders: 2004. p. 5.

9. Kawser CA, 2007, Chairman, Department of Paediatrics, BSMMU, Personal communication.

10. Roseberg S, Fujiara D. Epidemiology of Paediatric tumours of the nervous system according to WHO 2000 classification:a report of 1,195 cases from a single institution. Chils nerv Syst 2005; 21:940-944.

11. Farwell JR, Dohrmann GJ, Flannery JT. Central nervous system tumors in children. Cancer 1977; 40: 3123-32.

12. Rosai AJ. Ackerman's Surgical Pathology, $8^{\text {th }}$ edition. Mosby;1995. p. 2227-414.

13. Robison LL, Neglia JP, Mertens A. Epidemiology and etiology of childhood cancer. In: DJ Fernbach, TJ Vietti, editors. Clinical Paediatric Oncology, 4th Edition. St. Louis: CV Mosby; 1991. p. 11-28.

14. Preston-Martin S, Staples M, Farrugia H . Primary tumours of the brain ,cranial nerves and cranial meninges in Victoria,Australia.19821990: Pattern of incidence and survival, Neuroepidemiology 1993; 12: 270-9. 